Dieses Dokument ist eine Zweitveröffentlichung (Postprint) /

This is a self-archiving document (postprint):

Romina Kühn, Mandy Korzetz, Thomas Schlegel

User strategies for mobile device-based interactions to prevent shoulder surfing

Erstveröffentlichung in / First published in:

MUM '19: Proceedings of the 18th International Conference on Mobile and Ubiquitous Multimedia, Pisa, 2019. ACM Digital Library, Artikel-Nr. 43. ISBN 978-1-4503-7624-2

DOI: $\underline{\text { https://doi.org/10.1145/3365610.3368412 }}$

Diese Version ist verfügbar / This version is available on:

https://nbn-resolving.org/urn:nbn:de:bsz:14-qucosa2-709944 


\section{User Strategies for Mobile Device-Based Interactions to Prevent Shoulder Surfing}

\author{
Romina Kühn \\ TU Dresden \\ Dresden, Germany \\ romina.kuehn@tu-dresden.de
}

\author{
Mandy Korzetz \\ TU Dresden \\ Dresden, Germany \\ mandy.korzetz@tu-dresden.de
}

\author{
Thomas Schlegel \\ University of Applied Sciences \\ Karlsruhe, Germany \\ thomas.schlegel@hs-karlsruhe.de
}

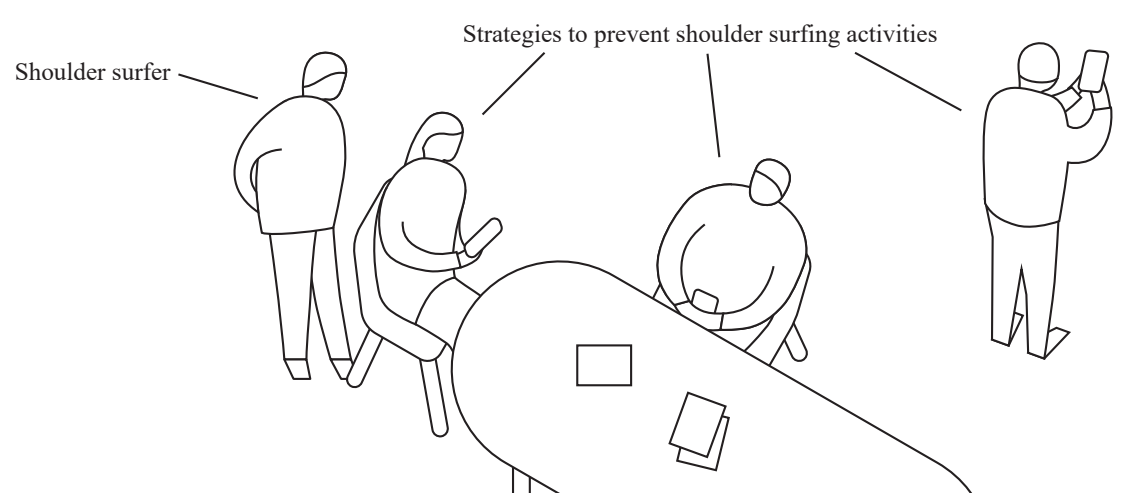

Figure 1: Shoulder surfing in a collocated collaboration scenario and some examples of user strategies to prevent it.

\begin{abstract}
Shoulder surfing, also known as visual hacking, is the activity of obtaining information from or about others by observing visual content of displays that actually should be kept secret, such as PINs, passwords, or private text messages. Approaches that address shoulder surfing on mobile devices mainly focus on ways to recognize observers or to complicate visual presentations for them from the system's perspective. However, users also have developed their own strategies to keep their input secret. With this work, we contribute an investigation of strategies to prevent shoulder surfing from the users' perspective. We performed a user study and observed 32 participants while interacting with smartphones using different kinds of eyes-free device-based interaction techniques. We identified several strategies that users had to prevent shoulder surfing. These strategies help us to develop effective ways to design useful interactions that overcome shoulder surfing issues.
\end{abstract}

\section{CCS CONCEPTS}

- Human-centered computing $\rightarrow$ Computer supported cooperative work.

\section{KEYWORDS}

Shoulder Surfing; Mobile Devices; Collocated Collaboration.

Permission to make digital or hard copies of part or all of this work for personal or classroom use is granted without fee provided that copies are not made or distributed for profit or commercial advantage and that copies bear this notice and the full citation on the first page. Copyrights for third-party components of this work must be honored

For all other uses, contact the owner/author(s).

MUM 2019, November 26-29, 2019, Pisa, Italy

(c) 2019 Copyright held by the owner/author(s).

ACM ISBN 978-1-4503-7624-2/19/11.

https://doi.org/10.1145/3365610.3368412
ACM Reference Format:

Romina Kühn, Mandy Korzetz, and Thomas Schlegel. 2019. User Strategies for Mobile Device-Based Interactions to Prevent Shoulder Surfing. In MUM 2019: 18th International Conference on Mobile and Ubiquitous Multimedia (MUM 2019), November 26-29, 2019, Pisa, Italy. ACM, New York, NY, USA, 5 pages. https://doi.org/10.1145/3365610.3368412

\section{INTRODUCTION}

Shoulder surfing is a common issue in public space, for example, while using the mobile device in a bus but also in work settings, for example, in open-plan offices. Even in several collaborative activities [17] in group work shoulder surfing occurs [12,16]. While the observation mainly concerns the visual content of a display, such as PINS, passwords, or private text messages, also the way of entering content can be observed. This includes input patterns, e.g. for unlocking the smartphone screen, as well as mobile-based interactions, e.g. touching devices with each other for connecting [11] or bumping devices to expand the view area [9]. Mobile-based interactions, as described by Korzetz et al. [14], are becoming more and more usual for concrete smartphone functionality, e.g. switching between front and rear camera of a Google Pixel device by twisting the phone twice or placing the smartphone face down on a flat surface to mute an incoming call. They do not need a look at the screen. However, such kinds of interactions are often very exposed and can be recognized and hence interpreted easily by observers.

With an increasing number of such mobile-based interaction techniques, we investigate ways to support users in avoiding shoulder surfing activities on their mobile devices, especially smartphones. On the system's side, there are several ways to obstruct observers from recognizing the input. Either mobile-based interactions need to be unobtrusive themselves, e.g. squeezing the device 
as introduced with the Google Pixel 2 phone, or they need to provide mechanisms to keep an input secret, e.g. back-of-device interactions $[5,6,19]$. Furthermore, there are recognition options using the front camera of the smartphone to detect observers [3].

However, there are also different user strategies to avoid shoulder surfing that users apply naturally. We investigated these strategies by performing a user study with 32 participants. With the results, we contribute a new perspective on shoulder surfing avoidance. In the following sections, we briefly describe related work that addresses different approaches to avoid or detect shoulder surfing activities. Further on, we present the user study we performed and the derived results. We conclude with highlighting the most important findings that can be used for further design of shoulder surfer prevented technology.

\section{RELATED WORK}

There is a wide range of contributions that address shoulder surfing on mobile devices (e.g. [2, 4, 12, 18, 21, 24]) and various strategies to overcome this problem. The majority of approaches focuses on password or PIN input and provides visual representations that are difficult to interpret as external person. For example, von Zezschwitz et al. [23] describe their approach SwiPIN where each number of a password or PIN is represented by a certain swipe gesture. Instead of tapping a button, users need to swipe in a given direction that represents a concrete number. Thus, shoulder surfers could not identify the particular number as easy as before. Gugenheimer et al. [8] present ColorSnake, a visual representation of PINs. To enter a PIN, users trace one given path out of several paths. Although, the path can be observed it is very difficult for an observer to identify the right path. Chen et al. [3] do not only focus on password or PIN input but they also address the visual presentation of a mobile device's screen. They use knowledge of human vision and optical systems to change the screen content by blending it into the background to hide it from shoulder surfers.

Some approaches use particularly innovative or unobtrusive interaction techniques to make it more difficult for observers to recognize input. De Luca et al. [5] present a back-of-device interaction for mobile device authentication. Because the input occurs at the backside of the device, shoulder surfers standing behind a user cannot see the input (see Fig. 1). Tafreshi et al. [22] present TiltPass, a gesture-based interaction technique that uses a tilt gesture as a shoulder surfer resistant password input for mobile devices. The password consists of different tilt gestures in different directions. Kühn et al. $[15,16]$ provide several device-based interactions for anonymous voting and rating that are eyes-free to perform.

There are also approaches that raise awareness for being observed. For example, Ali et al. [1] investigated shoulder surfing on mobile devices in public space. They provide an Android app (iAlert) that captures the surrounding of mobile phone users and detects people that are looking at the user's screen. The application alerts the user in case another person is looking at the screen of the mobile device. While they focus on the technology side of the detection, Saad et al. [20] evaluated different ways of communicating current shoulder surfing activities to the users.

Eiband et al. [7] investigated shoulder surfing in the wild and performed a user study where shoulder surfers reported on their motivation and feelings to observe other people. Additionally, users described their feelings and their acting when they realized they were observed. Although, Eiband et al. list different kinds of user actions after they realized they were observed, concrete user strategies to prevent shoulder surfing in the first place are not mentioned. Khan et al. [13] evaluated defense and attacker strategies and found that the most common defense strategy is to face away the display from the attackers sight. Again, the defense strategy was applied after the user was observed. We aim at closing this gap with our research by investigating proactive user strategies that aim at preventing shoulder surfing before it occurs.

\section{USER STUDY}

To investigate user strategies to prevent shoulder surfing, we utilized four concrete types of mobile-based interaction techniques that meet the definition of Korzetz et al. [14]: Tilt to Vote as a movement-based interaction, Fingerprint as back-of-device interaction, Draw to Vote as touch-based interaction, and Multitouch as multitouch interaction [15]. All four interaction techniques address collocated collaborative settings. Using these different types of mobile device-based interactions, we wanted to find out how users avoid shoulder surfing especially when there is no need to look at the screen of a mobile device [14]. Furthermore, we wanted to see what users do and how they reflect on their own explicit strategies. We therefore decided to use questionnaires as well as video records for further analysis. We performed the following user study.

\subsection{Participants}

We asked 32 participants ( 12 female) from age 18 to $51(M=31.2$, $\mathrm{SD}=6.4$ ) personally or via e-mail to participate in our user study. About two third of the participants were academics recruited from the university. All users were familiar with smartphone usage. We had 8 groups with 4 people each. Each participant was asked to act as shoulder surfer to trigger such activities within a group.

\subsection{Apparatus}

We provided a mobile Android application that included the four above-mentioned interaction techniques and a draw function. The participants received the task to draw small images for a fictional exhibition, share them with the other group members, and assess all created images. With this task, the participants should be motivated to keep their input secret as they did not want to show how they assessed. Consequently, they had to avoid the shoulder surfing activities of other group members. The four interaction techniques were used either to vote on the images whether they should be included in the fictional exhibition or to rate them on a scale from one to five. The movement-based interaction Tilt to Vote was triggered by tilting the mobile device along the $\mathrm{x}$ - or $\mathrm{y}$-axis. Draw to Vote as touch-based interaction used sequential touch inputs (one or two strokes) on the display, whereas Multitouch detected one to five synchronous touch inputs on the multitouch display. Finally, Fingerprint worked as back-of-device interaction where each recognized fingerprint in a short time period was counted as one rating point. Additionally, the Android application included a questionnaire which the participants had to complete after using the different device-based interaction techniques. 
User Strategies to Prevent Shoulder Surfing

\begin{tabular}{|c|c|c|c|c|c|}
\hline Interaction & $\begin{array}{l}0 \\
0 \\
0 \\
0 \\
0 \\
0 \\
0\end{array}$ & $\begin{array}{l}\frac{0}{0} \\
0 \\
0 \\
0 \\
0 \\
0 \\
0\end{array}$ & 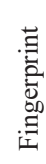 & 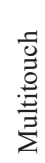 & $\sum$ \\
\hline Do nothing specific & 12 & 12 & 14 & 11 & 49 \\
\hline Hide device & 17 & 13 & 15 & 15 & 60 \\
\hline Observe surrounding & 1 & 0 & 1 & 2 & 4 \\
\hline Distract observer & 0 & 0 & 1 & 1 & 2 \\
\hline Vary way of entering & 2 & 7 & 1 & 3 & 13 \\
\hline
\end{tabular}

Figure 2: Total number of mentions of user strategies coming from the questionnaire.

\subsection{Procedure}

The study sessions were performed in a lab environment. After the participants arrived in the lab, they received detailed information concerning the global procedure of the study. We emphasized the focus on shoulder surfing activities and encouraged the participants to keep their input secret. We prepared the mobile application on four mobile phones which the participants could choose. Furthermore, we prepared a square table where the participants were allowed to take a seat but did not have to. They were explicitly allowed to walk freely within the lab. Additionally, a study leader was present the whole time and each session was recorded for retrospective analysis. First, the participants started with an introduction to become familiar with the four interaction techniques. Then, they performed the given task and shared their created images digitally. The images were assessed four times in total because participants were asked to use each interaction. After each interaction the participants completed a questionnaire concerning their own reflection on the strategies they used to avoid shoulder surfers. To conclude a session, we asked for final feedback in a semi-structured interview.

\section{FINDINGS AND DISCUSSION}

We analyzed the results from the questionnaires and the recorded videos. Furthermore, we interpreted the data and gained interesting insights into the strategies of users to prevent shoulder surfing.

\subsection{Results from Questionnaires}

After using each interaction techniques, we asked the participants what they did to keep their vote or rate secret. Participants answered with short descriptions of what they did. Then, we categorized the results in 5 categories. The first category is that participants did nothing specific. Participants stated clearly that it was sometimes not necessary to do something against shoulder surfing because they had the feeling that interactions were unobtrusive enough As second category, we identified users hid the device. Participants mainly used the table to hid the device but also body parts such as hands or legs. Ten times, participants did not specify how they hid their device. The third category comprises that participants observed the surrounding to find out whether they were observed or not. If not, then they rated or voted. Participants also distracted observers
MUM 2019, November 26-29, 2019, Pisa, Italy

\begin{tabular}{|c|c|c|c|c|c|}
\hline Interaction & $\begin{array}{l}\stackrel{0}{0} \\
\stackrel{0}{0} \\
\stackrel{0}{ \pm} \\
\pm=\end{array}$ & 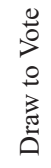 & 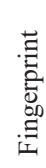 & 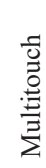 & 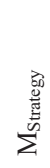 \\
\hline Do nothing specific & 2.7 & 4.3 & 3.7 & 2.7 & 3.4 \\
\hline Hide device & 1.9 & 4.0 & 3.1 & 3.5 & 3.1 \\
\hline Observe surrounding & 3.0 & - & 3.0 & 3.0 & 3.0 \\
\hline Distract observer & - & - & 1.0 & 3.0 & 2.0 \\
\hline Vary way of entering & 1.5 & 3.9 & 2.0 & 4.3 & 2.9 \\
\hline $\mathrm{M}_{\text {Interaction }}$ & 2.3 & 4.1 & 2.6 & 3.3 & \\
\hline
\end{tabular}

Figure 3: Mean values (M) from the 5-point Likert scale concerning the effort of keeping input secret $(5=$ little effort).

by making noises or movements so that it was not clear to observers how users rated. We identified the distraction as fourth category. That participants varied the way of entering is the fifth category. This category mainly comprises the speed of the input which was increased or decreased by the users to disguise the real input. Figure 2 shows the total numbers of strategies the participants used.

We further asked the participants to state how much effort it was for them to keep the input secret on a 5-point Likert scale ( 5 = best). From the questionnaire, we found that the effort depended both on the interaction technique as well as the user strategy. Figure 3 presents the mean values of the questionnaires' results. Both touch interactions (Draw to Vote and Multitouch) were rated as overall effortless in keeping input secret. In contrast, the movement-based interaction Tilt to Vote was rated with higher effort of keeping input secret. The back-of-device interaction Fingerprint received good ratings when hiding the device, doing nothing, and observing the surrounding but worse ratings for varying the way of entering and distracting observers. When comparing the different user strategies, hide device, observe, and vary entering received similar ratings for the mean values. In contrast, distracting required higher effort. Overall, as expected, doing nothing specific was rated with least effort $(M=3.4)$. However, from the questionnaires we could not find reasons that led to the devaluation of this category. Therefore, we continued to evaluate the observations we made.

\subsection{Results from Observations and Interviews}

After analyzing the questionnaires, we took the video records for further analysis. Overall, we investigated about 240 minutes of data. Our aim was to validate the findings from the questionnaires. Moreover, we wanted to substantiate the category do nothing specific.

First of all, we observed that the participants sat closely to each other around the table while performing the task. When it came to voting and rating they increased the distance to other participants by reclining on the chairs. At the same time, they pulled the devices towards the body to decrease the distance and started observing the surrounding. On the one hand, this behavior led to higher focus of the participants on the devices and their individual task. In terms of collaboration, the participants switched from close collaboration to 


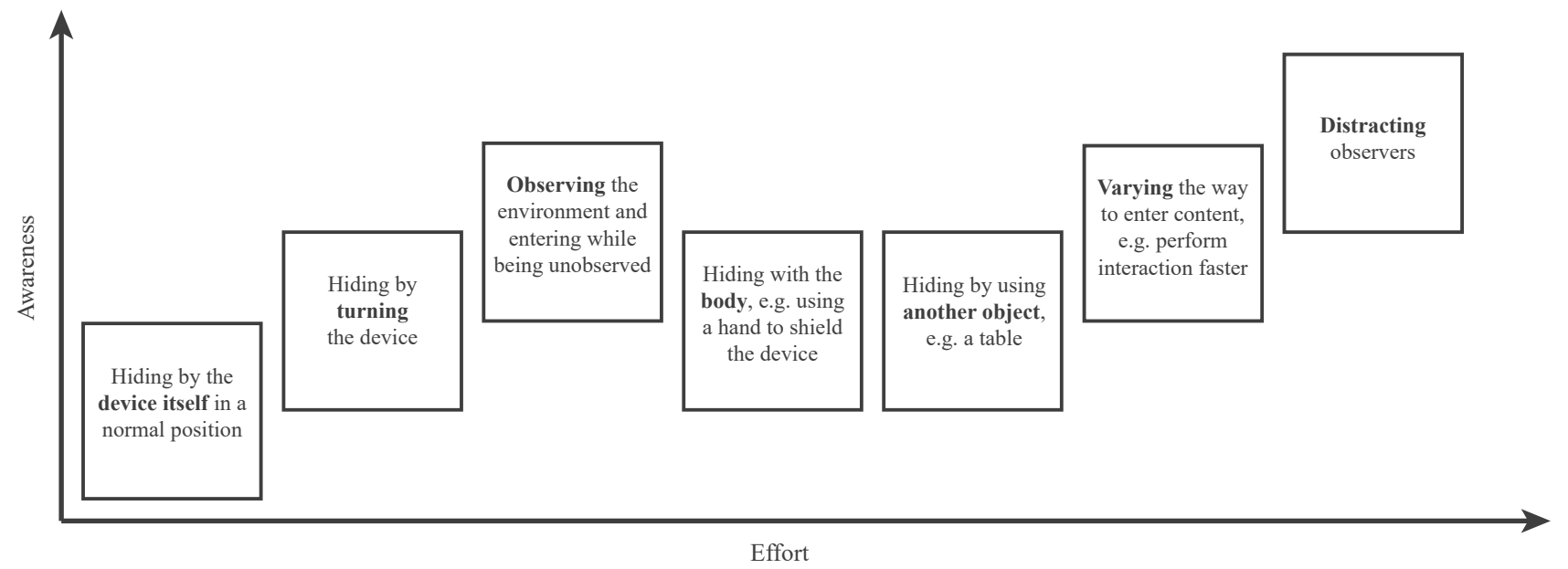

Figure 4: User strategies to prevent shoulder surfing.

loose collaboration [10]. On the other hand, shoulder surfers were triggered to observe because of the obvious movement.

We had a closer look at participants that stated to do nothing specific for avoiding shoulder surfers. We found that these participants often acted unconsciously with different strategies that we already described. Most commonly, they observed the surrounding while interacting with the device. They also changed their position to hide the device with their own body. According to these observations, both strategies (observing and hiding) seem to be very intuitive.

Furthermore, we identified four hiding strategies within the user study. The first strategy was hiding by the device itself, e.g. by using a back-of-device interaction. Participants used this strategy both consciously and unconsciously. Turning the device was the second way of hiding and was mainly used similarly to a back-of-device interaction. As a third way, participants shielded the device with their own body, e.g. a hand or the forearm. Lastly, participants hid their device with an object. In our study, participants often held their device under the table to hide it.

\subsection{Discussion}

From the results of our user study, we derived several concrete user strategies to prevent shoulder surfing as presented in Figure 4. We classified the user strategies into two dimensions: the effort of keeping input secret and the awareness of different strategies. Effort includes the need of moving as well as the need of changing focus from the task or interaction to, for example, observing the surrounding or distracting observers. Awareness is about user intention, intuitiveness, and concreteness of actions that need to be performed to keep an input secret. We arranged the observed strategies within these dimensions building on the recordings and interviews. The types of hiding devices with the device itself were the most intuitive and effortless strategies as we found from the results. Also observing the surrounding was identified as intuitive and effortless. However, from the questionnaires we assess them a little worse than hiding due to the number of times it was mentioned. Varying the way of entering content generated more effort depending on the variation. Similarly, distracting observers produced higher effort and needed conscious handling. Comparing our results with the results of Eiband et al. [7], we found that hiding and varying the way of entering were also proactive reactions after being observed not only to prevent shoulder surfing.

\subsection{Limitations and Future Work}

The user strategies we observed are based on mobile device-based interaction techniques. When investigating user strategies for conventional multitouch interactions the results could vary, especially, because device-based interactions do not need the display as visual representation of content. Therefore, we expect higher effort and greater need for awareness for user strategies to prevent shoulder surfing. Furthermore, we investigated four out of several more device-based interactions according to Korzetz et al. [14]. Although we suspect similar results and strategies for other mobile devicebased interactions, further investigations should be make.

\section{CONCLUSION}

In this paper, we described several user strategies to prevent shoulder surfing activities while using mobile device-based interactions in group settings. We identified seven strategies by conducting a user study with 32 participants. They comprise observing the surrounding while interacting, distracting observers, varying the way to interact, and hiding the device in different ways. Furthermore, we classified the identified strategies by the effort it takes and by the user awareness it needs. Hiding by the device itself and observing the surrounding were assessed best. The study results are the basis for further investigations concerning ways to prevent shoulder surfing on mobile devices. Our findings contribute to the open research gap of strategies from a user's perspective.

\section{ACKNOWLEDGMENTS}

The European Social Fund (ESF) and the Free State of Saxony have funded this work within the project CyPhyMan (100268299). 


\section{REFERENCES}

[1] Mohammed Eunus Ali, Anika Anwar, Ishrat Ahmed, Tanzima Hashem, Lars Kulik, and Egemen Tanin. 2014. Protecting Mobile Users from Visual Privacy Attacks. In Proceedings of the 2014 ACM International foint Conference on Pervasive and Ubiquitous Computing: Adjunct Publication (UbiComp '14 Adjunct). ACM, New York, NY, USA, 1-4. https://doi.org/10.1145/2638728.2638788

[2] Robert Biddle, Sonia Chiasson, and P.C. Van Oorschot. 2012. Graphical Passwords: Learning from the First Twelve Years. ACM Comput. Surv. 44, 4, Article 19 (Sept. 2012), 41 pages. https://doi.org/10.1145/2333112.2333114

[3] Chun-Yu Chen, Bo-Yao Lin, Junding Wang, and Kang G. Shin. 2019. Keep Others from Peeking at Your Mobile Device Screen!. In The 25th Annual International Conference on Mobile Computing and Networking (MobiCom '19). ACM, New York, NY, USA, Article 22, 16 pages. https://doi.org/10.1145/3300061.3300119

[4] Ashley Colley, Tobias Seitz, Tuomas Lappalainen, Matthias Kranz, and Jonna Häkkilä. 2016. Extending the Touchscreen Pattern Lock Mechanism with Duplicated and Temporal Codes. Advances in Human-Computer Interaction 2016 (2016), 11. https://doi.org/10.1155/2016/8762892

[5] Alexander De Luca, Marian Harbach, Emanuel von Zezschwitz, Max-Emanuel Maurer, Bernhard Ewald Slawik, Heinrich Hussmann, and Matthew Smith. 2014 Now You See Me, Now You Don'T: Protecting Smartphone Authentication from Shoulder Surfers. In Proceedings of the 32Nd Annual ACM Conference on Human Factors in Computing Systems (CHI '14). ACM, New York, NY, USA, 2937-2946. https://doi.org/10.1145/2556288.2557097

[6] Alexander De Luca, Emanuel von Zezschwitz, Ngo Dieu Huong Nguyen, MaxEmanuel Maurer, Elisa Rubegni, Marcello Paolo Scipioni, and Marc Langheinrich. 2013. Back-of-device Authentication on Smartphones. In Proceedings of the SIGCHI Conference on Human Factors in Computing Systems (CHI '13). ACM, New York, NY, USA, 2389-2398. https://doi.org/10.1145/2470654.2481330

[7] Malin Eiband, Mohamed Khamis, Emanuel von Zezschwitz, Heinrich Hussmann, and Florian Alt. 2017. Understanding Shoulder Surfing in the Wild: Stories from Users and Observers. In Proceedings of the 2017 CHI Conference on Human Factors in Computing Systems (CHI '17). ACM, New York, NY, USA, 4254-4265. https://doi.org/10.1145/3025453.3025636

[8] Jan Gugenheimer, Alexander De Luca, Hayato Hess, Stefan Karg, Dennis Wolf, and Enrico Rukzio. 2015. ColorSnakes: Using Colored Decoys to Secure Authentication in Sensitive Contexts. In Proceedings of the 17th International Conference on Human-Computer Interaction with Mobile Devices and Services (MobileHCI '15). ACM, New York, NY, USA, 274-283. https://doi.org/10.1145/2785830.2785834

[9] Ken Hinckley. 2003. Synchronous gestures for multiple persons and computers. In Proceedings of the 16th annual ACM symposium on User interface software and technology, Unknown(Ed.). ACM, 149-158. https://doi.org/10.1145/964696.964713

[10] Petra Isenberg, Danyel Fisher, Sharoda A. Paul, Meredith Ringel Morris, Kor Inkpen, and Mary Czerwinski. 2012. Co-located Collaborative Visual Analytics around a Tabletop Display. IEEE Transactions on Visualization and Computer Graphics 18, 5 (2012), 689-702. https://doi.org/10.1109/TVCG.2011.287

[11] Tero Jokela and Andrés Lucero. 2015. FlexiGroups: binding mobile devices for collaborative interactions in medium-sized groups with device touch. In Proceedings of the 16th ACM International Conference on Human-Computer Interaction with Mobile Devices and Services, Aaron Quigley, Sara Diamond, Pourang Irani, and Sriram Subramanian (Eds.). ACM, 369-378. https://doi.org/10.1145/2628363.2628376

[12] Mohamed Khamis, Linda Bandelow, Stina Schick, Dario Casadevall, Andreas Bulling, and Florian Alt. 2017. They Are All After You: Investigating the Viability of a Threat Model That Involves Multiple Shoulder Surfers. In Proceedings of the 16th International Conference on Mobile and Ubiquitous Multimedia (MUM '17). ACM, New York, NY, USA, 31-35. https://doi.org/10.1145/3152832.3152851

[13] Hassan Khan, Urs Hengartner, and Daniel Vogel. 2018. Evaluating Attack and Defense Strategies for Smartphone PIN Shoulder Surfing. In Proceedings of the 2018 CHI Conference on Human Factors in Computing Systems (CHI '18). ACM, New York, NY, USA, Article 164, 10 pages. https://doi.org/10.1145/3173574.3173738

[14] Mandy Korzetz, Romina Kühn, and Thomas Schlegel. 2019. Turn It, Pour It, Twist It: A Model for Designing Mobile Device-Based Interactions. In Proceedings of the 5th International ACM In-Cooperation HCI and UX Conference (CHIuXiD'19). ACM, New York, NY, USA, 20-23. https://doi.org/10.1145/3328243.3328246

[15] Romina Kühn, Mandy Korzetz, Lukas Büschel, Franz-Wilhelm Schumann, and Thomas Schlegel. 2016. Device-Based Interactions for Anonymous Voting and Rating with Mobile Devices in Collaborative Scenarios. In MUM '16: Proceedings of the 15th International Conference on Mobile and Ubiquitous Multimedia. 315-317.

[16] Romina Kühn, Mandy Korzetz, Franz-Wilhelm Schumann, Lukas Büschel, and Thomas Schlegel. 2019. Vote-for-It: Investigating Mobile Device-Based Interaction Techniques for Collocated Anonymous Voting and Rating. In Human-Computer Interaction - INTERACT 2019. 18.

[17] Romina Kühn and Thomas Schlegel. 2018. Mixed-Focus Collaboration Activities for Designing Mobile Interactions. In MobileHCI '18: Proceedings of the 20th International Conference on Human-Computer Interaction with Mobile Devices and Services. 71-78.

[18] Mun-Kyu Lee. 2014. Security Notions and Advanced Method for Human ShoulderSurfing Resistant PIN-Entry. Trans. Info. For. Sec. 9, 4 (April 2014), 695-708. https://doi.org/10.1109/TIFS.2014.2307671

[19] Luis A. Leiva and Alejandro Català. 2014. BoD Taps: An Improved Back-ofdevice Authentication Technique on Smartphones. In Proceedings of the 16th International Conference on Human-computer Interaction with Mobile Devices \&\#38; Services (MobileHCI '14). ACM, New York, NY, USA, 63-66. https://doi.org/ $10.1145 / 2628363.2628372$

[20] Alia Saad, Michael Chukwu, and Stefan Schneegass. 2018. Communicating Shoulder Surfing Attacks to Users. In Proceedings of the 17th International Conference on Mobile and Ubiquitous Multimedia (MUM 2018). ACM, New York, NY, USA, 147-152. https://doi.org/10.1145/3282894.3282919

[21] Florian Schaub, Ruben Deyhle, and Michael Weber. 2012. Password Entry Usability and Shoulder Surfing Susceptibility on Different Smartphone Platforms. In Proceedings of the 11th International Conference on Mobile and Ubiquitous Multimedia (MUM '12). ACM, New York, NY, USA, Article 13, 10 pages. https://doi.org/10.1145/2406367.2406384

[22] Amir E. Sarabadani Tafreshi, Sara C. Sarabadani Tafreshi, and Amirehsan Sarabadani Tafreshi. 2017. TiltPass: Using Device Tilts As an Authentication Method. In Proceedings of the 2017 ACM International Conference on Interactive Surfaces and Spaces (ISS '17). ACM, New York, NY, USA, 378-383. https://doi.org/10.1145/3132272.3134112

[23] Emanuel von Zezschwitz, Alexander De Luca, Bruno Brunkow, and Heinrich Hussmann. 2015. SwiPIN: Fast and Secure PIN-Entry on Smartphones. In Proceedings of the 33rd Annual ACM Conference on Human Factors in Computing Systems (CHI '15). ACM, New York, NY, USA, 1403-1406. https://doi.org/10.1145/ 2702123.2702212

[24] Oliver Wiese and Volker Roth. 2016. See You Next Time: A Model for Modern Shoulder Surfers. In Proceedings of the 18th International Conference on HumanComputer Interaction with Mobile Devices and Services (MobileHCI '16). ACM, New York, NY, USA, 453-464. https://doi.org/10.1145/2935334.2935388 